\title{
Adenobronchial fistula secondary to tuberculosis in an immunocompetent adult patient
}

\author{
Claudia Janeth Madrid Carbajal, ${ }_{1}^{1}$ Encarnación Nava Tomás, ${ }^{2}$ Miguel Ariza Prota, ${ }_{1}$ \\ Ana Isabel Enríquez Rodríguez, ${ }^{1}$ Marta García Clemente ${ }^{1}$
}

'Department of Respiratory Medicine, Hospital Universitario Central de Asturias, Oviedo, Spain

${ }^{2}$ Department of Radiology Medicine, Hospital Universitario Central de Asturias, Oviedo, Spain

\section{Correspondence to}

Claudia Janeth Madrid Carbajal, claudiamadrid9@hotmail.com

Accepted 16 October 2018

\section{DESCRIPTION}

A 72-year-old patient, retired miner, with no history of interest, which comes for an irritative cough with dark expectoration of 9 months of evolution. On physical examination, the patient was haemodynamically stable, afebrile and without notable alterations. No alterations were observed in the blood analysis and chest X-ray. A sputum microbiological study was performed observing resistant alcohol-acid bacilli, with positive Xpert Mycobacterium tuberculosis (MTB)/Rifampicin (RIF) study and culture of mycobacteria with MTB isolate sensitive to all first-line drugs. A chest CT scan showed calcified mediastinal and bilateral hilar adenopathies, a bilateral micronodular interstitial pattern suggestive of pneumoconiosis and an increase in pseudonodular density with a bronchogram on right interior lobe (RIL) suggestive of an infectious process. An air cavity of subcarinal localisation with fistulisation to both main bronchi is observed in relation to adenopathy with necrosis (figure 1A). A bronchoscopy was performed in which a fistulous orifice with thick discharge communicating through the subcarinal region with a fistula to the medial wall of the left main bronchus was observed in the medial wall of the right main bronchus (RMB) (figure 1B-D). Treatment was started with four drugs rifampicin, isoniazid, pyrazinamide and ethambutol with improvement of the clinical picture and compared with previous chest CT, fistulised cavity disappearance was observed in both main bronchi, with minimal fistulous tract persisting through the RMB. In addition, a bilateral micronodular interstitial pattern was observed in relation to pneumoconiosis given the professional background. There are no other pulmonary parenchymal changes that suggest an intercurrent infectious process (figure 2). 


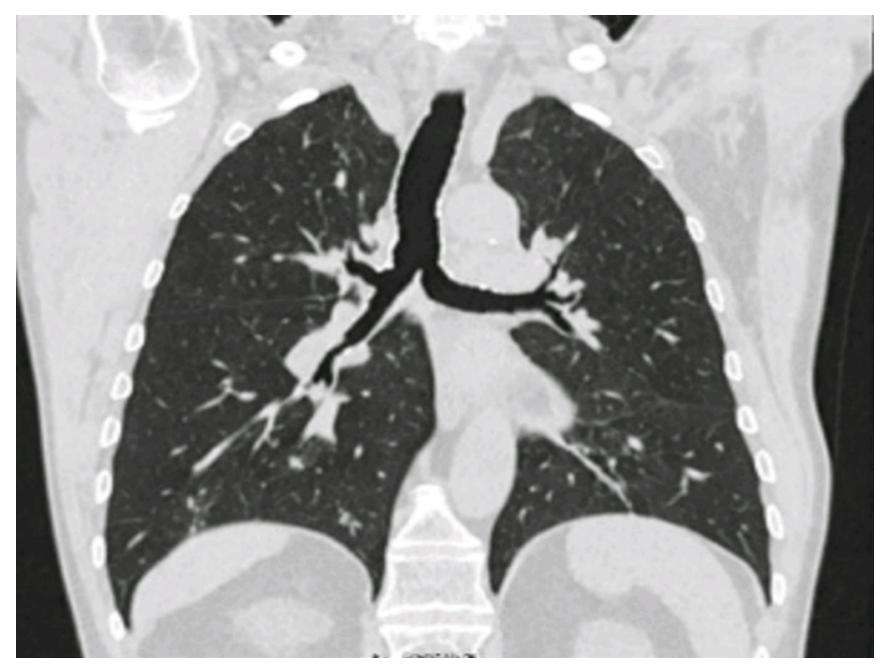

Figure 2 CT scan of the thorax after 4 months of treatment. Resolution of the fistulised mediastinal cavity was observed in both main bronchi, persisting a minimal fistulous trajectory with RMB origin. In addition, a bilateral micronodular interstitial pattern was observed in relation to pneumoconiosis given the professional background. There are no other pulmonary parenchymal changes that suggest an intercurrent infectious process. RMB, right main bronchus.

Different infection routes have been described, such as the spread of MTB from adenopathies to the bronchial wall through an adenobronchial fistula, this being more frequent in the paediatric population due to the weakness in the bronchial walls and the small diameter of the respiratory tract. ${ }^{1}$ The lymph nodes infected by MTB can erode adjacent structures such as oesophagus, heart, aorta and respiratory tract through inflammation with underlying necrosis and as a consequence fistulisation as in our case.

Bae et al described a case of adenobronchial fistulisation in an adult patient with immunosuppression due to lupus. ${ }^{2}$ It is important to mention the value of chest CT in the diagnosis of this condition. In a study by Park et al, the tomographic characteristics were described in seven patients with active pulmonary tuberculosis and adenobronchial fistula, concluding that the fistula occurs as a complication of extensive pulmonary tuberculosis with involvement of more than four lobes, occurring mainly in patients older than 72 years old and mainly involving the right bronchial side. ${ }^{3}$ In our case there is only involvement of RIL and the involvement was of both main bronchi in an immunocompetent patient. The value of bronchoscopy is essential to confirm the diagnosis, although there may be cases of fistula not easily visible due to mucosal oedema, which is why chest CT is recommended beforehand.

In conclusion, adenobronchial fistulisation due to tuberculosis is an exceptional complication in adult patients without immunosuppression.

\section{Learning points}

- To conclude we can say that the adenobronchial fistulae due to tuberculosis is an exceptional complication in adult patients without immunosuppression.

- To highlight the value of chest CT specially in patients with normal chest X-ray and the need to perform sputum baciloscopy in patients with long-term dry cough without explanation.

Contributors CJMC and ENT: manuscript preparation and review of literature, concepción and design, analysis, final approval of the version published. Agreement to be accountable for the article and to ensure that all questions regarding the accuracy or integrity of the article are investigated and resolved. MAP: manuscript preparation and literature review, analysis, final approval of the version published. Agreement to be accountable for the article and to ensure that all questions regarding the accuracy or integrity of the article are investigated and resolved. AIE: manuscript preparation, literature review and for getting patient's consent form, analysis, final approval of the version published. Agreement to be accountable for the article and to ensure that all questions regarding the accuracy or integrity of the article are investigated and resolved. MGC: manuscript preparation, literature review and clinical case supervision.

Funding The authors have not declared a specific grant for this research from any funding agency in the public, commercial or not-for-profit sectors.

Competing interests None declared.

Patient consent Obtained.

Provenance and peer review Not commissioned; externally peer reviewed.

\section{REFERENCES}

1 Marchiori E, Francisco FA, Zanetti G, et al. Lymphobronchial fistula: another complication associated with lymphobronchial tuberculosis in children. Pediatr Radiol 2013;43:252-3

2 Bae K, Jeon KN, Kim HC, et al. Tuberculosis presenting as isolated bronchonodal fistula in a patient with systemic lupus erythematosus. Medicine 2017;96:45:e8764.

3 Park SH, Jeon KN, Park MJ, et al. Tuberculous bronchonodal fistula in adult patients: CT findings. Jpn J Radio/ 2015;33:360-5.

Copyright 2018 BMJ Publishing Group. All rights reserved. For permission to reuse any of this content visit

http://group.bmj.com/group/rights-licensing/permissions.

BMJ Case Report Fellows may re-use this article for personal use and teaching without any further permission.

Become a Fellow of BMJ Case Reports today and you can:

- Submit as many cases as you like

- Enjoy fast sympathetic peer review and rapid publication of accepted articles

- Access all the published articles

- Re-use any of the published material for personal use and teaching without further permission

For information on Institutional Fellowships contact consortiasales@bmjgroup.com

Visit casereports.bmj.com for more articles like this and to become a Fellow 